\title{
The Galactic evolution of phosphorus *
}

\author{
E. Caffau ${ }^{\star \star, 1,2}$, P. Bonifacio ${ }^{2}$, R. Faraggiana ${ }^{3}$, and M. Steffen ${ }^{4,2}$ \\ ${ }^{1}$ Zentrum für Astronomie der Universität Heidelberg, Landessternwarte, Königstuhl 12, 69117 Heidelberg, Germany \\ e-mail: elisabetta.caffau@obspm.fr \\ 2 GEPI, Observatoire de Paris, CNRS, Université Paris Diderot, Place Jules Janssen, 92190 Meudon, France \\ 3 Università degli Studi di Trieste, via G.B. Tiepolo 11, 34143 Trieste, Italy \\ ${ }^{4}$ Leibniz-Institut für Astrophysik Potsdam, An der Sternwarte 16, 14482 Potsdam, Germany
}

Received 21 May 2011 / Accepted 28 June 2011

\section{ABSTRACT}

\begin{abstract}
Context. As a galaxy evolves, its chemical composition changes and the abundance ratios of different elements are powerful probes of the underlying evolutionary processes. Phosphorous is an element whose evolution has remained quite elusive until now, because it is difficult to detect in cool stars. The infrared weak P I lines of the multiplet 1 , at 1050-1082 nm, are the most reliable indicators of the presence of phosphorus. The availability of CRIRES at VLT has permitted access to this wavelength range in stellar spectra. Aims. We attempt to measure the phosphorus abundance of twenty cool stars in the Galactic disk.

Methods. The spectra are analysed with one-dimensional model-atmospheres computed in local thermodynamic equilibrium (LTE). The line formation computations are performed assuming LTE.

Results. The ratio of phosphorus to iron behaves similarly to sulphur, increasing towards lower metallicity stars. Its ratio with respect to sulphur is roughly constant and slightly larger than solar, $[\mathrm{P} / \mathrm{S}]=0.10 \pm 0.10$.

Conclusions. We succeed in taking an important step towards the understanding of the chemical evolution of phosphorus in the Galaxy. However, the observed rise in the $\mathrm{P} / \mathrm{Fe}$ abundance ratio is steeper than predicted by Galactic chemical evolution model developed by Kobayashi and collaborators. Phosphorus appears to evolve differently from the light odd-Z elements sodium and aluminium. The constant value of $[\mathrm{P} / \mathrm{S}]$ with metallicity implies that $\mathrm{P}$ production is insensitive to the neutron excess, thus processes other than neutron captures operate. We suggest that proton captures on ${ }^{30} \mathrm{Si}$ and $\alpha$ captures on ${ }^{27} \mathrm{Al}$ are possibilities to investigate. We see no clear distinction between our results for stars with planets and stars without any detected planet.
\end{abstract}

Key words. stars: abundances - stars: atmospheres - line: formation - Galaxy: evolution - Galaxy: disk - radiative transfer

\section{Introduction}

Phosphorus is an abundant element in the Universe, and in the solar photosphere it is among the top twenty most abundant elements. In the periodic table of elements, phosphorus, with $Z=15$, is in the same group as nitrogen, lying between silicon $(Z=14)$ and sulphur $(Z=16)$. Silicon is a well-studied element, and its abundance is easily measured in photospheres of stars of type $\mathrm{F}, \mathrm{G}$, or $\mathrm{K}$, by analysing the Si I lines. In the last fifteen years, sulphur has been systematically investigated by several groups that analysed the few multiplets of $S_{\text {I }}$ available in the observed spectra (for further details see Spite et al. 2011, and references therein). This is not the case for phosphorus, that, before this work, had never been analysed systematically in cool stars. The reason why was already given by Struve (1930): no P I line is available in the "ordinary" range of the observed spectra of stars of spectral type F, G, or K. Some P II and $\mathrm{P}$ III lines are observable in the spectra of B-type stars. In the UV spectrum, some lines of $\mathrm{P}_{\text {IV }}, \mathrm{P}_{\text {III, }}$ and $\mathrm{P}_{\text {II }}$ can be detected in hot stars. To study the variation in the phosphorous abundance over time we need to use long-lived stars of type F, G, or K. In the era of the high resolution spectrograph CRIRES-VLT, some

* Based on observations obtained with the CRIRES spectrograph at ESO-VLT Antu $8.2 \mathrm{~m}$ telescope at Paranal, Programme 386.D-0130, P.I. E. Caffau.

$\star \star$ Gliese fellow. lines of P I, the lines of Mult. 1, at 1050-1082 nm, can be observed. For the first time, it has thus become possible to trace the Galactic evolution of phosphorus.

One single stable isotope of phosphorus, ${ }^{31} \mathrm{P}$, is believed to be formed via neutron capture, as for the parent nuclei ${ }^{29} \mathrm{Si}$ and ${ }^{30} \mathrm{Si}$, probably in the carbon and neon burning shells during the late stages of the evolution of massive stars. The ${ }^{31} \mathrm{P}$ produced in this way is then released by the explosion of these massive stars as type II SNe. Woosley \& Weaver (1995) expect no significant production of phosphorus during the explosive phases.

The handful of observations available of $\mathrm{P}$ in different stars was summarised in Caffau et al. (2007). Since then, Hubrig et al. (2009) have derived the P abundance in horizontal branch (HB) stars in globular clusters NGC 6397 and NGC 6752, where it appears to be strongly enhanced, by more than 2 dex and almost 3 dex, respectively. Chemical anomalies in HB stars are usually attributed to diffusion effects, but the current models by Michaud et al. (2008) do not seem to account for more than about 0.7 dex of the $\mathrm{P}$ enhancement in the photosphere of HB stars of similar temperature.

Meléndez et al. (2009) measured P in ten solar twins, although they do not state which lines they used. The lines of Mult. 1 were not available in their observations and we speculate that they used the strongest line of Mult. 2 at $979.6 \mathrm{~nm}$.

A result concerning $\mathrm{P}$ evolution that we find intriguing, is that of Sbordone et al. (2009), who found that the sulphur 
abundance in the globular cluster 47 Tuc displays a significant correlation with the $\mathrm{Na}$ abundance. The authors point out that $\mathrm{S}$ could be produced through proton capture reactions on $\mathrm{P}$ $\left({ }^{31} \mathrm{P}(p, \gamma)^{32} \mathrm{~S}\right)$. However, $\mathrm{P}$ in the Sun is roughly $1.7 \mathrm{dex}$ less abundant than $\mathrm{S}$. If the same abundance ratio held elsewhere, it would be impossible to produce any significant amount of $S$ at the expense of $\mathrm{P}$. The $\mathrm{P}$ abundance has never been measured in stars of 47 Tuc.

In this paper, we use high-resolution infrared (IR) spectra, obtained with CRIRES at VLT, to analyse the behaviour of the $\mathrm{P}$ abundance as a function of metallicity in the Galactic disc. We selected a sample of stars from Caffau et al. (2005) for which the $\mathrm{S}$ abundance is known, to assess whether the proton capture on $\mathrm{P}$ is a viable production channel for $\mathrm{S}$. The sample spans a metallicity range of $1.2 \mathrm{dex},-1.0<[\mathrm{Fe} / \mathrm{H}]<+0.3$. Our future plan is to extend the sample of metal-poor stars, and to include metallicities lower than $[\mathrm{Fe} / \mathrm{H}]^{1}=-1$.

\section{Observed spectra}

The strongest of the $\mathrm{P}_{\mathrm{I}}$ lines $(1058.1569 \mathrm{~nm})$ has an equivalent width of about $2.2 \mathrm{pm}$ in the spectrum of the solar photosphere. In a solar temperature star, all the lines of Mult. 1 are hardly visible when the metallicity is below $[\mathrm{Fe} / \mathrm{H}]=-0.5$. They are of high excitation energy (see Table 1) and sensitive to temperature, hence become weaker in cooler stars. We wished to derive $\mathrm{P}$ abundance across as wide a range in metallicity as possible, to study the Galactic evolution of phosphorus. From the sample of Caffau et al. (2005), that is stars for which the abundance of sulphur was known, we selected 22 main-sequence or turn-off, $\mathrm{F}$ to early G-type stars. We gave preference to warmer stars, and tried to have a significant number of stars with detected planets. All the stars of the sample happen to be bright, in the range 3.3-7 mag in J. At the VLT-Antu $8 \mathrm{~m}$ telescope, these stars can be observed in twilight and under very poor weather conditions.

All our spectra were observed by nodding along the slit. The slit width was 0.2 , providing a spectral resolution $R=100000$; the adaptive optics correction was computed on axis, using the target star itself. Twenty of our stars have been observed in service mode in the program 386.D-0130 (PI, E. Caffau). We used the setting centred at $1059.5 \mathrm{~nm}$ in order 54, to ensure that the four detectable lines of $\mathrm{P}_{\mathrm{I}}$ were positioned on detectors 2 and 3 , which are the most efficient. The two reddest lines of Mult. 1 (1068.1 and $1081.3 \mathrm{~nm}$ ), that are the weakest lines of the multiplet in the solar spectrum, fall outside the range of the CRIRES detectors. Our analysis was performed with the reduced spectra provided by ESO.

\section{Model atmospheres and atomic data}

We analysed four of the IR P I lines of Mult. 1. The atomic data we used, listed in Table 1, are from Berzinsh et al. (1997).

For each star, we computed a 1D-LTE model atmosphere with the code ATLAS 12 in its Linux version (Kurucz 2005; Sbordone et al. 2004; Sbordone 2005), with the parameters $\left(T_{\text {eff }} / \log g /[\mathrm{Fe} / \mathrm{H}]\right)$ from Caffau et al. (2005) and references therein, used to derive the sulphur abundance. The only exception is HD 1461 for which we choose the atmospheric parameters of Sousa et al. (2008) and the S abundance of González Hernández et al. (2010) (see Sect. 5 for details). This choice was driven by the need to make a consistent comparison between phosphorus and sulphur. The ATLAS 12 models were

${ }_{1[\mathrm{X} / \mathrm{H}]}=\log \left(N_{\mathrm{X}} / N_{\mathrm{H}}\right)-\log \left(N_{\mathrm{X}} / N_{\mathrm{H}}\right)_{\odot}$ and $[\mathrm{X} / \mathrm{Y}]=[\mathrm{X} / \mathrm{H}]-[\mathrm{Y} / \mathrm{H}]$.
Table 1. Infrared phosphorus lines analysed in this work.

\begin{tabular}{lccc}
\hline \hline $\begin{array}{l}\lambda \\
{[\mathrm{nm}]}\end{array}$ & Transition & $\begin{array}{l}E_{\text {low }} \\
{[\mathrm{eV}]}\end{array}$ & $\log g f$ \\
\hline 1051.1584 & $4 \mathrm{~s}^{4} \mathrm{P}_{1 / 2}-4 \mathrm{p}^{4} \mathrm{D}_{3 / 2}^{0}$ & 6.94 & -0.13 \\
1052.9522 & $4 \mathrm{~s}^{4} \mathrm{P}_{3 / 2}-4 \mathrm{p}^{4} \mathrm{D}_{5 / 2}^{0}$ & 6.95 & 0.24 \\
1058.1569 & $4 \mathrm{~s}^{4} \mathrm{P}_{5 / 2}-4 \mathrm{p}^{4} \mathrm{D}_{7 / 2}^{0}$ & 6.99 & 0.45 \\
1059.6900 & $4 \mathrm{~s}^{4} \mathrm{P}_{1 / 2}-4 \mathrm{p}^{4} \mathrm{D}_{1 / 2}^{0}$ & 6.94 & -0.21 \\
\hline
\end{tabular}

computed with a mixing length parameter of $\alpha_{\mathrm{MLT}}=0.5$ and the overshooting option switched off.

\section{Analysis}

We measured the equivalent width $(E W)$ of the $\mathrm{P}_{\mathrm{I}}$ lines, by using the $\operatorname{iraf}^{2}$ task splot. The lines are weak: the strongest line has $\log (E W / \lambda)=-5.36$, but the majority of the lines have $\log (E W / \lambda) \leq-5.5$, and a Gaussian profile is a good approximation for the line profile fitting. The $\mathrm{P}_{\mathrm{I}}$ line at $1051 \mathrm{~nm}$ is, for several stars, contaminated by telluric absorption (see upper-left panel of Fig. 1). When possible, we measured the $E W$ of the P I line taking into account the presence of the telluric contamination, otherwise we rejected the line. Two of the lines (1052 and $1058 \mathrm{~nm}$ ) are blended with a $\mathrm{Ni}$ I and $\mathrm{Si}$ I line, respectively. According to the strength of the lines and the stellar $V \sin i$, we fitted the PI line alone, or took into account the close-by line at the same time, using the deblending option of splot. We used the code WIDTH (Kurucz 1993, 2005; Castelli 2005) to derive the $\mathrm{P}$ abundance from the $E W$. The results are listed in Table 2, where the $\mathrm{P}$ abundance is given in the last but one column together with the line-to-line scatter. Figure 1 shows the four $\mathrm{P}_{\mathrm{I}}$ lines for the star HD 13555.

We looked at the effect that an uncertainty in temperature and gravity has on the $\mathrm{P}$ abundance determination. The computations refer to the model $6112 \mathrm{~K} / 4.34 /+0.16$. As a typical value for the uncertainty in the temperature and the gravity, we took $\Delta T_{\text {eff }}=100 \mathrm{~K}$ and $\Delta \log g=0.2 \mathrm{dex}$, respectively. Looking at the literature, we found that $T_{\text {eff }}$ and $\log g$ in these stars usually do not differ between different authors by more than the uncertainties we assume here. The change in temperature $\Delta T_{\text {eff }}= \pm 100$ induces $\Delta[\mathrm{P} / \mathrm{H}]={ }_{+0.04}^{-0.03}$ dex. For the gravity, $\Delta \log g= \pm 0.2$ dex induces $\Delta[\mathrm{P} / \mathrm{H}]={ }_{-0.05}^{+0.06}$ dex. The choice of the mixing length parameter has very little influence on the determination of the $\mathrm{P}$ abundance. A change in the mixing length parameter, from 0.5 to 1.5 , increases the $\mathrm{P}$ abundance of $+0.0045 \mathrm{dex}$, which is, for the quality of the data we analyse here, absolutely negligible. We assumed the systematic uncertainty in the $\mathrm{P}$ abundance to be 0.1 dex, by linearly adding the contribution due to a change in effective temperature and in gravity. We neglect the contribution due to a change in the mixing length parameter. The statistical uncertainty due to the $E W$ measurement is negligible with respect to this systematic error.

To our knowledge, no study of the deviation from local thermal equilibrium for phosphorus has been published, and no P model-atom, necessary for this computation, seems to exist in the literature. Caffau et al. (2007) studied the effects that granulation has on the $\mathrm{P}$ abundance determination in the solar photosphere. These effects are very small $(0.02,0.03,0.04$, and 0.02

2 Image Reduction and Analysis Facility, written and supported by the IRAF programming group at the National Optical Astronomy Observatories (NOAO) in Tucson, Arizona. http://iraf.noao.edu/ 
Table 2. Stellar parameters and abundances of our program stars.

\begin{tabular}{|c|c|c|c|c|c|c|c|c|c|c|c|c|}
\hline Target & $\begin{array}{c}T_{\text {eff }} \\
\mathrm{K}\end{array}$ & $\log g$ & {$[\mathrm{Fe} / \mathrm{H}]$} & {$[\mathrm{S} / \mathrm{H}]$} & Ref. & $\begin{array}{c}S / N \\
\# 2 / \# 3\end{array}$ & $\begin{array}{c}E W[\mathrm{pm}] \\
1051.1\end{array}$ & $\begin{array}{c}E W[\mathrm{pm}] \\
1052.9\end{array}$ & $\begin{array}{c}E W[\mathrm{pm}] \\
1058.1\end{array}$ & $\begin{array}{c}E W[\mathrm{pm}] \\
1059.6\end{array}$ & {$[\mathrm{P} / \mathrm{H}]$} & Notes \\
\hline HD 1461 & 5765 & 4.38 & +0.19 & -0.05 & G10 & $180 / 150$ & & 2.00 & 2.70 & 0.95 & $+0.14 \pm 0.01$ & $\mathrm{P}$ \\
\hline HD 13555 & 6470 & 3.90 & -0.27 & -0.25 & T02 & $400 / 300$ & 0.80 & 1.80 & 2.90 & 0.84 & $-0.28 \pm 0.03$ & \\
\hline HD 25704 & 5792 & 4.20 & -0.91 & -0.71 & $\mathrm{C} 05$ & $300 / 150$ & 0.30 & 0.65 & 1.20 & 0.30 & $-0.55 \pm 0.05$ & \\
\hline HD 33256 & 6440 & 3.99 & -0.37 & -0.30 & T02 & $350 / 250$ & & 1.90 & 3.20 & 1.00 & $-0.20 \pm 0.04$ & \\
\hline HD 69897 & 6227 & 4.20 & -0.50 & -0.34 & $\mathrm{C} 02$ & $120 / 50$ & 0.83 & 2.00 & 3.00 & & $-0.17 \pm 0.06$ & \\
\hline HD 74156 & 6112 & 4.34 & +0.16 & -0.13 & E04 & $250 / 90$ & 1.15 & 2.40 & 3.60 & 1.00 & $+0.05 \pm 0.04$ & $\mathrm{P}$ \\
\hline HD 75289 & 6143 & 4.42 & +0.28 & -0.03 & E04 & $180 / 80$ & 1.50 & 2.70 & 3.60 & & $+0.16 \pm 0.01$ & $\mathrm{P}$ \\
\hline HD 84117 & 6167 & 4.35 & -0.03 & -0.10 & E04 & $150 / 70$ & & 2.40 & 4.00 & & $+0.09 \pm 0.07$ & \\
\hline HD 91324 & 6123 & 3.95 & -0.60 & -0.49 & $\mathrm{C} 02$ & $300 / 140$ & & 1.60 & 2.40 & & $-0.30 \pm 0.02$ & \\
\hline HD 94388 & 6379 & 3.96 & +0.07 & +0.12 & $\mathrm{C} 02$ & $200 / 120$ & 1.40 & 3.40 & 4.40 & & $+0.03 \pm 0.07$ & V \\
\hline HD 120136 & 6339 & 4.19 & +0.23 & +0.05 & E04 & $250 / 170$ & 1.90 & 4.60 & & 1.90 & $+0.26 \pm 0.08$ & $\mathrm{P}, \mathrm{V}$ \\
\hline HD 139211 & 6231 & 4.12 & -0.26 & -0.16 & $\mathrm{C} 02$ & $300 / 240$ & 1.40 & 3.20 & 3.50 & 1.40 & $+0.03 \pm 0.05$ & \\
\hline HD 207129 & 5910 & 4.42 & 0.00 & -0.05 & E04 & $400 / 250$ & 0.75 & 1.60 & 2.10 & 0.70 & $-0.09 \pm 0.02$ & $\mathrm{~T}$ \\
\hline HD 207978 & 6400 & 4.03 & -0.63 & -0.53 & T02 & $300 / 170$ & 0.69 & 1.40 & 1.60 & & $-0.47 \pm 0.05$ & \\
\hline HD 209458 & 6117 & 4.48 & +0.02 & -0.20 & E04 & $200 / 100$ & & 2.00 & 3.00 & & $0.00 \pm 0.03$ & $\mathrm{P}$ \\
\hline HD 213240 & 5984 & 4.25 & +0.17 & -0.10 & E04 & $300 / 180$ & 1.40 & 2.75 & 3.60 & 1.20 & $+0.15 \pm 0.01$ & $\mathrm{P}$ \\
\hline HD 215648 & 6158 & 3.96 & -0.24 & -0.19 & $\mathrm{C} 02$ & $220 / 100$ & 0.96 & 2.10 & 2.80 & & $-0.19 \pm 0.02$ & \\
\hline HD 216385 & 6300 & 3.91 & -0.27 & -0.18 & T02 & $400 / 180$ & 1.10 & 2.40 & 3.00 & 0.90 & $-0.18 \pm 0.02$ & \\
\hline HD 216435 & 5938 & 4.12 & +0.24 & +0.10 & E04 & $250 / 130$ & 1.80 & 3.30 & 4.10 & 1.50 & $+0.26 \pm 0.02$ & $\mathrm{P}$ \\
\hline HD 222368 & 6178 & 4.08 & -0.13 & -0.07 & $\mathrm{C} 02$ & $280 / 130$ & 1.30 & 2.40 & 3.50 & 1.20 & $-0.03 \pm 0.02$ & $\mathrm{~V}$ \\
\hline
\end{tabular}

Notes. The column "Ref." refers to the reference for the stellar parameters and [S/H], and corresponds to, G10: González Hernández et al. (2010); T02: Takada-Hidai et al. (2002); C05: Caffau et al. (2005); C02: Chen et al. (2002); E07: Ecuvillon et al. (2004).

The column "Notes" reports on the characteristics of the stars; P: star with planets, V: variable star; T: pre-main sequence T-Tauri.

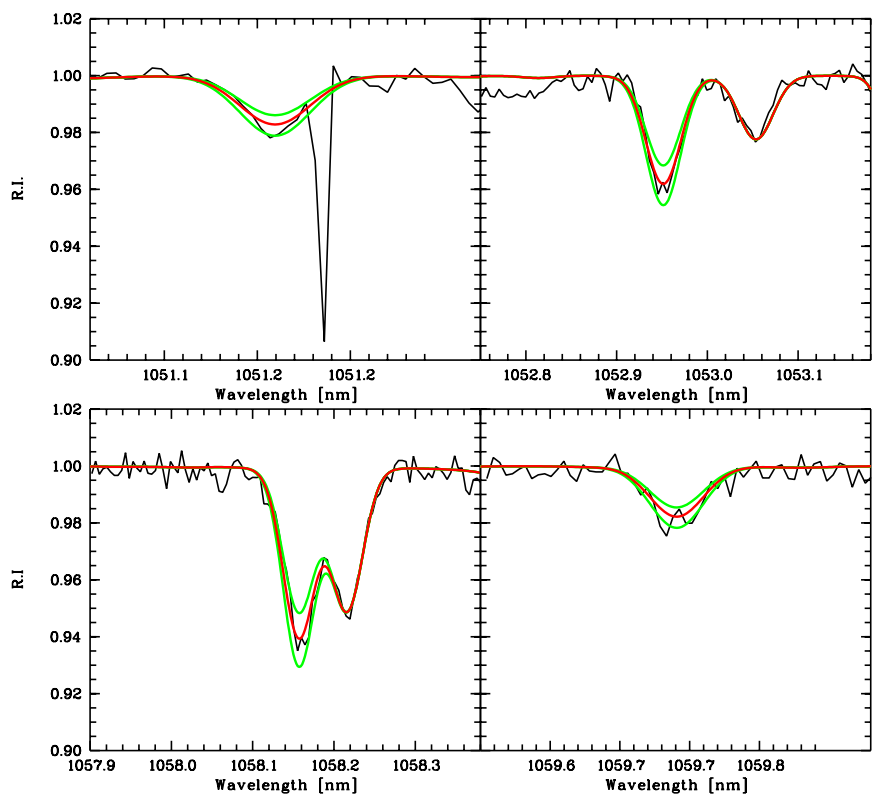

Fig. 1. The four Pi lines are here shown in the case of HD 13555. The observed spectrum (solid black) is compared to the synthetic profile (solid red) with the $\mathrm{P}$ abundance derived from the $E W$ measurement. Also shown are the synthetic profiles obtained by changing the $\mathrm{P}$ abundance by \pm 0.1 dex (solid green). The telluric absorption affecting the $1051 \mathrm{pm}$ line is clearly visible in the upper-left panel.

for the four P I lines we analyse here) compared to our error estimate, and we decided not to apply any three-dimensional (hereafter 3D) correction. The stars of our sample span a small range in effective temperature and gravity and our 3D-model grid (Ludwig et al. 2009) is very coarse. An application of the 3D corrections interpolated from the small number of available models would result in tiny shifts in the abundances, much smaller than the associated error of \pm 0.1 dex.

\section{Discussion}

In Fig. 2, $[\mathrm{P} / \mathrm{Fe}]$ is plotted versus the metallicity, $[\mathrm{Fe} / \mathrm{H}]$. The $[\mathrm{P} / \mathrm{Fe}]$ ratio has a behaviour similar to that of an $\alpha$-element. We can clearly see $[\mathrm{P} / \mathrm{Fe}]$ increases as $[\mathrm{Fe} / \mathrm{H}]$ decreases at metallicities as low as solar metallicity, $[\mathrm{P} / \mathrm{Fe}]$ is close to zero for solar metallicity stars, and increases as metallicity decreases.

For the light odd- $Z$ elements it is generally assumed that the main production channel is neutron capture (Burbidge et al. 1957). If this is the case, one can expect that the production of $\mathrm{P}$ is sensitive to the neutron excess ${ }^{3} \eta$. Since the neutron excess decreases with decreasing metallicity one can naively expect that the ratios of $\mathrm{P}$ to elements that are insensitive to $\eta$, such as $\mathrm{S}$ or $\mathrm{Fe}$, should decrease with decreasing metallicity. The increase in $[\mathrm{P} / \mathrm{Fe}]$ with decreasing metallicity contradicts this simple expectation. It should however be noted that in the metallicity range covered by our study neither $\mathrm{Na}$ nor $\mathrm{Al}$, the nearest odd- $Z$ elements, display a decrease in their ratios to iron with decreasing metallicity (Gehren et al. 2004; Shi et al. 2004; Gehren et al. 2006), but instead remain constant.

Owing to the small number of metal-poor stars, it is unclear whether $[\mathrm{P} / \mathrm{Fe}]$ reaches a plateau at about $[\mathrm{Fe} / \mathrm{H}] \approx-0.5$, or continues increasing. More data at metallicity around -1.0 would be very useful to clarify this interesting question. The observed data in Fig. 2 are compared to the model of P evolution in the Galaxy by Kobayashi et al. (2006) (solid line). The model seems to qualitatively reproduce the observed behaviour,

3 The neutron excess is defined by Arnett (1971) as: $\eta=$ $\left(n_{\mathrm{n}}-n_{\mathrm{p}}\right) /\left(n_{\mathrm{n}}+n_{\mathrm{p}}\right)$, where $n_{\mathrm{n}}$ represents the total number of neutrons per unit mass and $n_{\mathrm{p}}$ the number of protons per unit mass. 


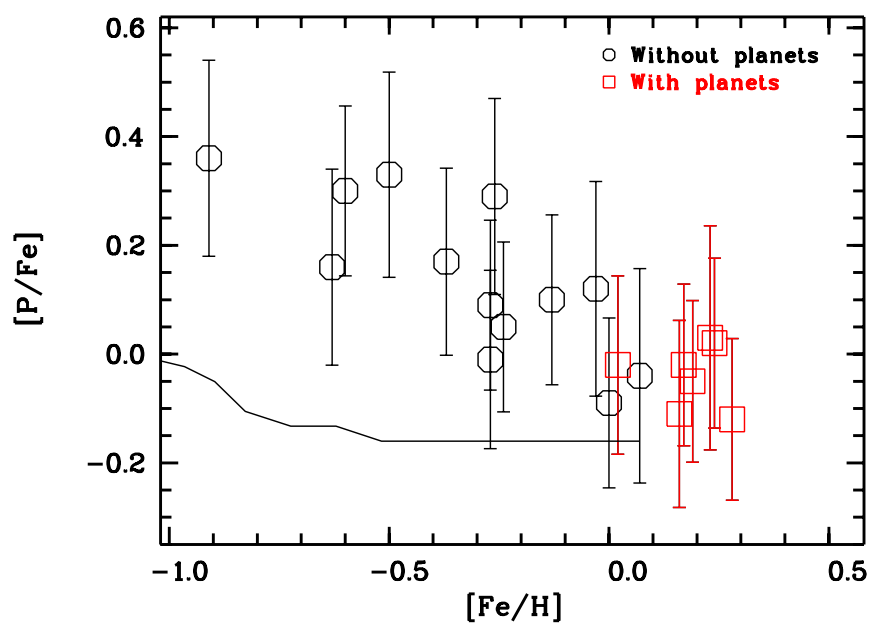

Fig. 2. $[\mathrm{P} / \mathrm{Fe}]$ as a function of the metallicity, $[\mathrm{Fe} / \mathrm{H}]$. The dimension of the symbols reflects the average line-to-line scatter of 0.045 dex. The error bars are the sum under quadrature of the uncertainties of $\mathrm{P}$ (the linear sum of line-to-line scatter and the systematic uncertainty) and of $\mathrm{Fe}$, the latter assumed to be 0.1 dex for all stars. Stars known to have planets (red squares) have different symbols from stars without known planet (black hexagons). A model of Galactic evolution of P (Kobayashi et al. 2006) is added (solid line) for comparison to the observed data.

although quantitatively the increase in $[\mathrm{P} / \mathrm{Fe}]$ is stronger than predicted and already starts at solar metallicity.

In our sample, seven stars have detected planets and are plotted as squares in Fig. 2. We see no difference in the behaviour in the $[\mathrm{P} / \mathrm{Fe}]$ trend between the sample with and without planets. One should always bear in mind that for the stars "without planets" it cannot be excluded that they harbour undetected planets. We note, however, that all of the stars "without planets" in our sample, except for HD 215648 and HD 207978 have several high precision radial velocity points measured with the HARPS spectrograph at the ESO $3.6 \mathrm{~m}$ telescope. Given that, based on these radial velocity measurements, no planet has been reported, short-period massive planets ("hot Jupiters") should be excluded for these stars.

In Fig. 3, $[\mathrm{S} / \mathrm{Fe}]$ versus (vs.) $[\mathrm{Fe} / \mathrm{H}]$ is shown for the same sample of stars. There is a tiny difference between stars with and without planets. The difference is reflected in the $[\mathrm{P} / \mathrm{S}]$ vs. $[\mathrm{Fe} / \mathrm{H}]$ plot in Fig. 4. Phosphorus and sulphur behave similarly when effective temperature and gravity of the model atmosphere are changed. We computed the systematic uncertainties in $\mathrm{S}$ with the models we used for the $\mathrm{P}$ analysis, and assume that the systematic error in the $[\mathrm{P} / \mathrm{S}]$ ratio is given by the difference between the (logarithmic) systematic errors in $\mathrm{P}$ and $\mathrm{S}$. The stars with planets are the most metal-rich ones in the sample, and have a lower sulphur abundances than those without planets. The trend of $[\mathrm{P} / \mathrm{S}]$ with the metallicity is flat, and has a large scatter (see Fig. 4). The average value of this "plateau" is $[\mathrm{P} / \mathrm{S}]=0.10 \pm 0.10$. As noted above when discussing the $[\mathrm{P} / \mathrm{Fe}]$ ratio, this "plateau" in $[\mathrm{P} / \mathrm{S}]$ is surprising. In the same metallicity range, the ratios of either $\mathrm{Na}$ or $\mathrm{Al}$ to the $\alpha$ element $\mathrm{Mg}$ decrease with decreasing metallicity (Gehren et al. 2006).

For the seven stars with known planets, we find $\langle[\mathrm{P} / \mathrm{S}]\rangle=$ $0.15 \pm 0.10$, while for the stars without known planets $\langle[\mathrm{P} / \mathrm{S}]\rangle=$ $0.07 \pm 0.10$. This finding is not statistically significant, because the two values agree to within one $\sigma$. We also note that there is hardly any overlap in metallicity between the stars with and without planets. Nevertheless, this difference is

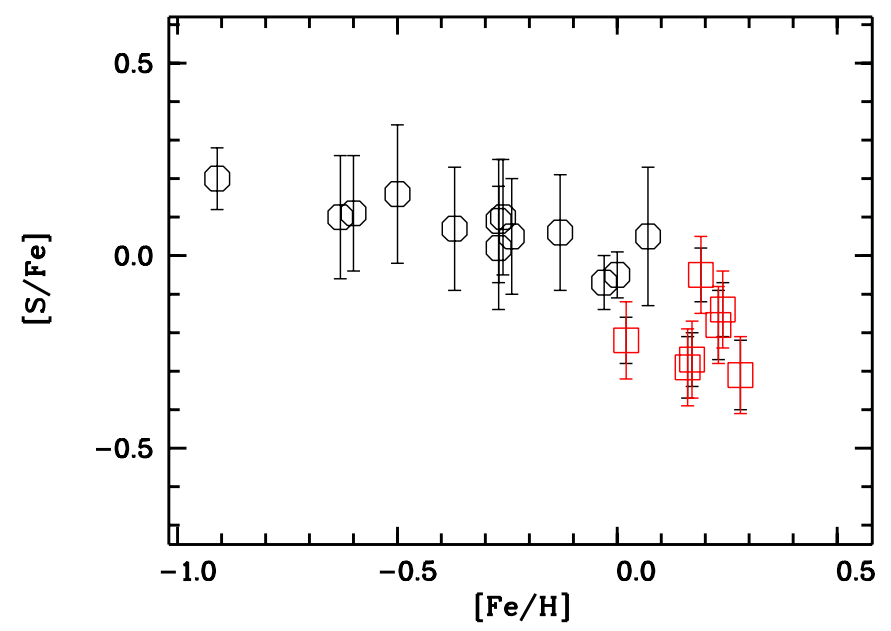

Fig. 3. $[\mathrm{S} / \mathrm{Fe}]$ as a function of the metallicity, $[\mathrm{Fe} / \mathrm{H}]$. The error bars indicate the uncertainties of $[\mathrm{Fe} / \mathrm{S}]$ as given in the related papers. The symbols have the same meaning as in Fig. 2.

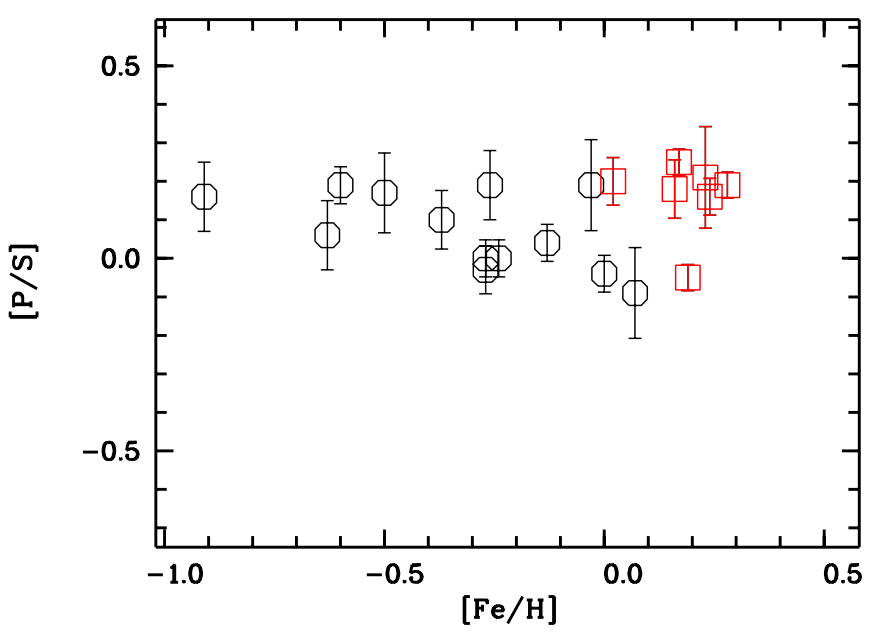

Fig. 4. $[\mathrm{P} / \mathrm{S}]$ as a function of the metallicity, $[\mathrm{Fe} / \mathrm{H}]$. Symbols are as in Fig. 2. The error bars are computed as the linear sum of the systematic error of $[\mathrm{P} / \mathrm{S}]$ (see text) and $\sqrt{2}$ times the $\mathrm{P}$ line-to-line scatter (assuming that random errors of $\mathrm{P}$ and $\mathrm{S}$ are of similar size).

puzzling and caused by the evolution in the abundance of sulphur, not phosphorous.

The two suspected binary stars (HD 33256, HD 69897) do not behave very differently from the complete sample. We can therefore conclude that the companion is either of the same spectral type or much fainter. In addition, the three stars classified as variable in the Simbad ${ }^{4}$ astronomical database (HD 94388, HD 120136, HD 222368) and the pre main-sequence star (HD 207129) do not display any difference from the other stars in the sample. For the three variable stars, the photometric variability of HD 94388 and HD 222368 was reported by Petit (1990). Later, however, these two stars were used as standards by several authors (e.g. HD 94388 for delta Scuti stars by Hintz et al. 1998; and HD 222368 for long-term variables by Manfroid et al. 1995). The slight variability of HD 120136 is studied by Hall et al. (2009), who confirm the long-term variability detected by Lockwood et al. (2007).

${ }^{4}$ http://simbad.u-strasbg.fr/simbad/ 
For HD 1461, we report in Table 2 the measurement of the sulphur abundance by González Hernández et al. (2010), who used the atmospheric parameters of Sousa et al. (2008).

Rivera et al. (2010) found a super-Earth on a shortperiod orbit (5.77 days) around HD 1461. This star, and its planet, has been the subject of an intense debate between González Hernández et al. (2010) and Ramírez et al. (2010). The debate revolves around the claim made by Meléndez et al. (2009) that the Sun has a peculiar chemical composition with respect to the average of solar-twins and solar analogs, being overabundant in volatile elements and under-abundant in refractory elements. Meléndez et al. (2009) proceed to identify the reason for this "peculiarity" is the presence of rocky planets, such as the Earth. This claim is supported by Ramírez et al. (2010), but rejected by González Hernández et al. (2010) who, in an independent analysis, do not confirm the results of Meléndez et al. (2009) and point out that HD 1461, hosting a super-Earth, has an abundance pattern that differs from what is claimed to be a signature of the presence of terrestrial planets by Meléndez et al. (2009) and Ramírez et al. (2010). This conclusion is contested by Ramírez et al. (2010), who claim, when restricting the analysis to refractory elements, that HD 1461 conforms to their claim. In this debate, the abundance of phosphorous provides data for a new element. In the sample of solar twins of Meléndez et al. (2009), the mean $[\mathrm{P} / \mathrm{Fe}]$ is -0.051 with a $\sigma$ of 0.063 , in excellent agreement with what we found in HD 1461 (-0.050), despite being a rocky planet host.

\section{Conclusions}

We have determined the phosphorus abundance in twenty stars of the Galactic disk that we observed with CRIRES at VLT. The metallicity of the sample extends over about 1.2 dex, providing a first insight into the evolution of phosphorus in the Galaxy. The only published model of the chemical evolution of the Galaxy that provides information about the evolution of phosphorus is that of Kobayashi et al. (2006). The model predicts correctly, from a qualitative point of view, the rise of the $\mathrm{P} / \mathrm{Fe}$ ratio with decreasing metallicity. The observed rise is, however, larger by almost a factor of two than the model's prediction, and lacks the "plateau" at metallicities $[\mathrm{Fe} / \mathrm{H}]>-0.5$. Our analysis indicates that phosphorus behaves in a similar way to an $\alpha$-element, such as sulphur. The model of Galactic chemical evolution described by Gibson et al. (2005) predicts a $\sim 0.2$ dex enhancement of the $[\mathrm{P} / \mathrm{Fe}]$ ratio over two dex in metallicity, from solar to -2.0 . The level of enhancement at low metallicity is compatible with what we observe, but no mention is made of the decrease in this ratio near solar metallicity.

We have found that the $[\mathrm{P} / \mathrm{S}]$ is roughly constant at a level of about 0.1 dex. However, the star-to-star scatter makes this value compatible with zero. This constant value implies that $\mathrm{P}$ and $\mathrm{S}$ are produced in the same amounts over the metallicity range considered by our investigation. The picture is different from what is found for $\mathrm{Na}$ or $\mathrm{Al}$ : both $[\mathrm{Na} / \mathrm{Mg}]$ and $[\mathrm{Al} / \mathrm{Mg}]$ decrease significantly with decreasing metallicity. A possible interpretation is that the $\mathrm{P}$ production is insensitive to the neutron excess $\eta$. If this is the case, then $\mathrm{P}$ should be produced by nuclear reactions other than neutron captures. Possibilities that should be explored are proton captures on ${ }^{30} \mathrm{Si}$ and $\alpha$ captures on ${ }^{27} \mathrm{Al}$. A corollary of this analysis is that, since in the Sun sulphur is about a factor of 30 more abundant than phosphorus, this ratio remains the same at all metallicities (in the range that we have examined). We therefore conclude that $\mathrm{S}$ production through proton captures on $\mathrm{P}$ is not a viable mechanism. Although the possibility of a large enhancement of $\mathrm{P}$ abundance in globular clusters cannot be ruled out, it seems unlikely.

It is interesting to compare our results to phosphorus abundances derived from measurements in damped Ly- $\alpha$ (DLA) systems at high redshift. These are gas-rich galaxies observed as absorption lines in the direction of QSOs. Molaro et al. (2001) measured the $\mathrm{P}$ abundance in the DLA at $z=3.3901$ towards QSO 0000-2620. This system has a very low metallicity and measurements of $[\mathrm{P} / \mathrm{H}]=-2.31,[\mathrm{P} / \mathrm{Fe}]=-0.27$, and $[\mathrm{P} / \mathrm{S}]=-0.40$. The ratios of $\mathrm{P}$ to iron and sulphur are clearly different from what those seen in Galactic disk stars. This may be due to the low metallicity of the system, suggesting that perhaps also in our Galaxy, the $[\mathrm{P} / \mathrm{Fe}]$ ratio starts at low values, increases until reaching a maximum, and thereafter decreases towards solar metallicity. This behaviour is predicted by the model of Kobayashi et al. (2006), although not shown in Fig. 2, which only extends down to $[\mathrm{Fe} / \mathrm{H}]=-1.0$. Another possibility is that this DLA galaxy has undergone a different chemical evolution, since it has $[\mathrm{S} / \mathrm{Fe}]=+0.13$, while at these metallicities $[\mathrm{S} / \mathrm{Fe}] \sim+0.4$ in the Galaxy (Caffau et al. 2005).

Fenner et al. (2004) determined an upper limit of $[\mathrm{P} / \mathrm{S}]<$ +0.01 in the DLA at $z=2.626$ towards FJ081240.6+320808 This system is more metal-rich $([\mathrm{O} / \mathrm{H}]=-0.44)$, thus probably of metallicity comparable to some of the stars in our sample. However, the $[\mathrm{P} / \mathrm{S}]$ ratio is slightly lower than what we observe. Although this DLA system may resemble the Milky Way disk, it has probably experienced a different chemical evolution.

Our investigation is the first step towards an understanding of the evolution of $\mathrm{P}$ in the Galaxy. It will be important to secure more data at metallicity $[\mathrm{Fe} / \mathrm{H}]=-1.0$ and lower, to understand whether the $[\mathrm{P} / \mathrm{Fe}]$ ratio flattens out, continues to rise, or finally decreases towards low metallicity. The good performance of the CRIRES spectrograph suggests that it will be possible to observe considerably fainter targets with adequate exposure times. A direct measurement of $\mathrm{P}$ in 47 Tuc or other globular clusters would allow us to probe the feasibility of $\mathrm{S}$ production through proton capture in these objects.

Acknowledgements. We acknowledge support from the Programme National de Physique Stellaire (PNPS) and the Programme National de Cosmologie et Galaxies (PNCG) of the Institut National de Sciences de l'Univers of CNRS.

\section{References}

Arnett, W. D. 1971, ApJ, 166, 153

Berzinsh, U., Svanberg, S., \& Biemont, E. 1997, A\&A, 326, 412

Burbidge, E. M., Burbidge, G. R., Fowler, W. A., \& Hoyle, F. 1957, Rev. Mod. Phys., 29, 547

Caffau, E., Bonifacio, P., Faraggiana, R., et al. 2005, A\&A, 441, 533

Caffau, E., Steffen, M., Sbordone, L., Ludwig, H.-G., \& Bonifacio, P. 2007, A\&A, 473, L9

Castelli, F. 2005, Mem. Soc. Astron. Ital. Suppl., 8, 44

Chen, Y. Q., Nissen, P. E., Zhao, G., \& Asplund, M. 2002, A\&A, 390, 225

Ecuvillon, A., Israelian, G., Santos, N. C., et al. 2004, A\&A, 426, 619

Fenner, Y., Prochaska, J. X., \& Gibson, B. K. 2004, ApJ, 606, 116

Gehren, T., Liang, Y. C., Shi, J. R., Zhang, H. W., \& Zhao, G. 2004, A\&A, 413, 1045

Gehren, T., Shi, J. R., Zhang, H. W., Zhao, G., \& Korn, A. J. 2006, A\&A, 451, 1065

Gibson, B. K., Fenner, Y., \& Kiessling, A. 2005, Nucl. Phys. A, 758, 259

González Hernández, J. I., Israelian, G., Santos, N. C., et al. 2010, ApJ, 720, 1592 
Hall, J. C., Henry, G. W., Lockwood, G. W., Skiff, B. A., \& Saar, S. H. 2009, AJ, 138,312

Hintz, M. L., Joner, M. D., \& Hintz, E. G. 1998, AJ, 116, 2993

Hubrig, S., Castelli, F., de Silva, G., et al. 2009, A\&A, 499, 865

Kobayashi, C., Umeda, H., Nomoto, K., Tominaga, N., \& Ohkubo, T. 2006, ApJ, 653,1145

Kurucz, R. 1993, SYNTHE Spectrum Synthesis Programs and Line Data, Kurucz CD-ROM, No. 18, Cambridge, Mass.: Smithsonian Astrophysical Observatory

Kurucz, R. L. 2005, Mem. Soc. Astron. Ital. Suppl., 8, 14

Lockwood, G. W., Skiff, B. A., Henry, G. W., et al. 2007, ApJS, 171, 260

Ludwig, H.-G., Caffau, E., Steffen, M., et al. 2009, Mem. Soc. Astron. Ital., 80, 711

Manfroid, J., Sterken, C., Cunow, B., et al. 1995, A\&AS, 109, 329

Meléndez, J., Asplund, M., Gustafsson, B., \& Yong, D. 2009, ApJ, 704, L66

Michaud, G., Richer, J., \& Richard, O. 2008, ApJ, 675, 1223
Molaro, P., Levshakov, S. A., D’Odorico, S., Bonifacio, P., \& Centurión, M. 2001, ApJ, 549, 90

Petit, M. 1990, A\&AS, 85, 971

Ramírez, I., Asplund, M., Baumann, P., Meléndez, J., \& Bensby, T. 2010, A\&A, 521, A33

Rivera, E. J., Butler, R. P., Vogt, S. S., et al. 2010, ApJ, 708, 1492

Sbordone, L. 2005, Mem. Soc. Astron. Ital. Suppl., 8, 61

Sbordone, L., Bonifacio, P., Castelli, F., \& Kurucz, R. L. 2004, Mem. Soc. Astron. Ital. Suppl., 5, 93

Sbordone, L., Limongi, M., Chieffi, A., et al. 2009, A\&A, 503, 121

Shi, J. R., Gehren, T., \& Zhao, G. 2004, A\&A, 423, 683

Sousa, S. G., Santos, N. C., Mayor, M., et al. 2008, A\&A, 487, 373

Spite, M., Caffau, E., Andrievsky, S. M., et al. 2011, A\&A, 528, A9

Struve, O. 1930, ApJ, 71, 150

Takada-Hidai, M., Takeda, Y., Sato, S., et al. 2002, ApJ, 573, 614

Woosley, S. E., \& Weaver, T. A. 1995, ApJS, 101, 181 